www.nature.com/jhg

\title{
A commentary on a novel splicing silencer generated by $D M D$ exon 45 deletion junction could explain upstream exon 44 skipping that modifies dystrophinopathy
}

\author{
Yukitoshi Ishikawa \\ Journal of Human Genetics (2014) 59, 419-420; doi:10.1038/jhg.2014.49; published online 3 July 2014
}

$\mathrm{D}$ uchenne and Becker muscular dystrophy (DMD/BMD) are allelic X-linked recessive neuromuscular disorder caused by dystrophin gene (MIM no.300377) mutations; ${ }^{1}$ two-thirds $(65-70 \%)$ of patients with DMD have large deletions or duplications, the other one-third have nonsense mutations, small deletions/insertions and splice site mutations. ${ }^{2}$ Genotype-phenotype correlation is well explained by the reading frame rule; ${ }^{3}$ out of frame type mutations by frameshift of one or few exons makes the premature termination of dystrophin protein, and inframe type mutation that the reading frame is preserved makes the defective protein.

To this time, deletion junction sequences in outliers to the reading frame rule had never been intensively investigated.

The article 'a novel splicing silencer generated by DMD exon 45 deletion junction could explain upstream exon 44 skipping that modifies dystrophinopathy' by Dwianingsih et al. ${ }^{4}$ describes that alternative splicing event due to a single exon deletion in the patient with DMD could modify the clinical severity; exon 45 deletion in a milder case than typical case with DMD generated a novel splicing silencer in intronic sequences of junction and induced the partial exon 44 skipping. In this article, the authors clearly elucidated the molecular basis in the index case by the fine genetic analysis.

In this article, I was so impressed in three points of view.

Y Ishikawa is at Department of Pediatrics, National Hospital Organization Yakumo Hospital, Yakumo, Japan E-mail: y-ishi@host.or.jp
At first, the authors had established a molecular analysis strategy for atypical patient with dystrophinopathy. Although the index case showed a single exon 45 deletion in genomic DNA, his clinical severity was milder compared to typical cases. The authors decided the analysis of mRNA in his muscle and found the partial exon 44 skipping that became in-frame deletion. Finally, the authors conducted the sequencing of the deletion junction by PCR walking and demonstrated the huge deletion ( $87 \mathrm{kp}: 72.6 \mathrm{~kb}+176 \mathrm{bp}+14.3 \mathrm{~kb}$ ). The authors had already reported several DMD/BMD patients with unique molecular basis, utilizing these RNA-based analysis (reverse transcription (RT)-PCR analysis and then sequencing of cDNA from biopsyed muscle sample). ${ }^{5-7}$ These strategy will be useful and more informative for understanding the molecular basis in such unique patients with dystrophinopathy.

In second aspect, the authors clearly identified the transcript derived from $\Delta 44 / 45$ dys which produced internally truncated dystrophin was estimated to be $6 \%$ of the normal level by real-time RT-PCR. This amount of transcript is sufficient to elongate the independent ambulation period to 18 years in the index case. This is a first report of phenotypic conversion of DMD to a milder case due to the exon 44 skipping. These findings represented that the minimum levels of in-frame mRNA restored by exon-skipping should be at least $6 \%$ and strongly support the theoretical mechanism in the recent gene therapy by exonskipping. ${ }^{8,9}$
Finally, the authors had established the useful system analyzing in vitro splicing activity using chimeric dsx (Drosophila melanogaster doublesex) pre-mRNA in HeLa cell nuclear extracts. ${ }^{7}$ This system can clearly detect splicing regulatory activity by inserting the target sequences downstream of the ASLV (avian sarcoma-leukosis virus) enhancer sequence. The authors tested $270 \mathrm{bp}$ sequence of the junction and identified a part of the newly created junction sequences joining introns 44 and 45 , which was similar to an octamer of FGFR2 gene that had acted as the splicing silencer. This evidence was further confirmed by inhibition of the splicing silencer activity of the junction sequence by treatment of the antisense oligonucleotides complementary to the $5^{\prime}$ or $3^{\prime}$ part of the junction sequence.

As closing remarks, this article first had described the newly created junction sequences by single exon 45 deletion that have been explored as splicing modulators and it acted as a splicing silencer that induced upstream exon 44 skipping. This is the first recognized mechanism of phenotypic conversion of DMD to a milder case. Therefore, these findings strongly encourage the disease-modifying approach for DMD by exon-skipping.

1 Koenig, M., Hoffman, E. P., Bertelson, C. J., Monaco, A. P., Feener, C. \& Kunkel, L. M. Complete cloning of the Duchenne muscular dystrophy (DMD) cDNA and preliminary genomic organization of the DMD gene in normal and affected individuals. Cell 50, 509-517 (1987)

2 Takeshima, Y., Yagi, M., Okizuka, Y., Awano, H., Zhang, Z., Yamauchi, Y. et al. Mutation spectrum of the dystrophin gene in 442 Duchenne/Becker muscular 
dystrophy cases from one Japanese referral center. J. Hum. Genet. 55, 379-388 (2010).

3 Monaco, A. P., Bertelson, C. J., Liechti-Gallati, S. Moser, H. \& Kunkel, L. M. An explanation for the phenotypic differences between patients bearing partial deletions of the DMD locus. Genomics 2, 90-95 (1988).

4 Dwianingsih, E. K., Malueka, R. G., Nishida, A. Itoh, K., Lee, T., Yagi, M. et al. A novel splicing silencer generated by $D M D$ exon 45 deletion junction could explain upstream exon 44 skipping that modifies dystrophinopathy. J. Hum. Genet. 59, 423-429 (2014).
5 Matsuo, M., Masumura, T., Nakajima, T., Kitoh, Y., Takumi, T., Nishio, H. et al. A very small frame-shifting deletion within exon 19 of the Duchenne muscular dystrophy gene. Biochem. Biophys. Res. Commun. 170, 963-967 (1990).

6 Matsuo, M., Masumura, T., Nishio, H., Nakajima, T., Kitoh, Y., Takumi, T. et al. Exon skipping during splicing of dystrophin mRNA precursor due to an intraexon deletion in the dystrophin gene of Duchenne muscular dystrophy Kobe. J. Clin. Invest. 87, 2127-2131 (1991).

7 Shiga, N., Takeshima, Y., Sakamoto, H., Inoue, K., Yokota, Y., Yokoyama, M. et al. Disruption of the splicing enhancer sequence within exon 27 of the dystrophin gene by a nonsense mutation induces partial skipping of the exon and is responsible for Becker muscular dystrophy. J. Clin. Invest. 100, 2204-2210 (1997).

8 Matsuo, M. Duchenne/Becker muscular dystrophy: from molecular diagnosis to gene therapy. Brain Dev. 18, 167-172 (1996).

9 Takeshima, Y., Wada, H., Yagi, M., Ishikawa, Y., Ishikawa, Y., Minami, R. et al. Oligonucleotides against a splicing enhancer sequence led to dystrophin production in muscle cells from a Duchenne muscular dystrophy patient. Brain Dev. 23, 788-790 (2001). 